\section{The brain barriers as therapeutic targets in neuroinflammation}

B. Engelhardt ${ }^{1}$

1 Theodor Kocher Institute, University of Bern, Bern, Switzerland

The central nervous system (CNS) is an immunologically privileged site to which access of circulating immune cells is tightly controlled by the endothelial blood-brain barrier (BBB) localized in CNS microvessels and the epithelial bloodcerebrospinal fluid barrier (BCSFB) within the choroid plexus and subsequently by the glia limitans. Due to the specialized structure of the brain barriers, immune cell entry into the CNS parenchyma involves two differently regulated steps: migration of immune cells across the BBB or BCSFB into the cerebrospinal fluid (CSF) drained spaces of the CNS, followed by progression across the glia limitans into the CNS parenchy- ma. With a focus on multiple sclerosis (MS) and stroke and their animal models I will explain the molecular mechanisms involved in immune cell trafficking across the brain barriers and explain the benefits and risks of therapeutic targeting of these mechanism in MS and stroke. As neuroinflammation is usually accompanied by BBB leakiness I will further discuss how therapeutic stabilization of barrier integrity might be beneficial in treating MS, especially at timepoints when antiinflammatory treatments fail.

Conflict of Interest: The author declares no conflict of interest.

\section{Bibliography}

Dol http://dx.doi.org/10.1055/s-0033-1346705

Drug Res 2013; 63, Suppl. 1: S5-S5

(c) Georg Thieme Verlag KG Stuttgart · New York .

ISSN 2194-9379 